\title{
Introduction to HICSS-54 Business Intelligence, Business Analytics and Big Data: Innovation, Deployment and Management Minitrack
}

\author{
Olivera Marjanovic \\ University of Technology Sydney \\ Australia \\ olivera.marjanovic@uts.edu.au
}

\author{
Barbara Dinter \\ Chemnitz University of Technology \\ Germany \\ barbara.dinter@wirtschaft.tu- \\ chemnitz.de
}

\author{
Thilini Ariyachandra \\ Xavier University \\ USA \\ ariyachandrat@xavier.edu
}

\begin{abstract}
Now in its $31^{\text {st }}$ year, this minitrack is the longest running HICSS minitrack related to business intelligence (BI), business analytics (BA) and more recently big data that continues to rise in relevance to both research and practice. Its long history of trailblazing research and an ongoing focus on the organizational issues, create a minitrack with a rich comprehensive view of contemporary challenges of $\mathrm{BI} / \mathrm{BA}$ and big data innovation, deployment and management.
\end{abstract}

This year's minitrack includes the following six research papers:

The opening paper by Rashed and Drews describes four pathways for designing and realizing data-driven business models (DDBMs). They include: i) a use case-centric; ii) technology-centric; iii) DDBM integration; and iv) DDBM startup pathways. The identified pathways enable practitioners to better navigate the challenging journey of implementing DDBM in their own contexts.

In the second paper, Kayser, Fruhwirth and Mueller use a structured literature review to investigate the existing classification approaches to data-driven innovation. The study emphasizes the importance of connecting different literature streams and developing a shared language across them.

Using a design science research method, Fadler and Legner propose a framework for enterprise-wide data and analytics governance. The framework is developed in collaboration with experts from five large corporations.

The fourth paper by Ramm, Kopf, Dinter, and Honigsberg explores the concept as well as the use and acceptance of storytelling in Business Intelligence (BI). The authors used a systematic literature review to derive a morphological box of storytelling in BI. Then, they adapted the Unified Theory of Acceptance and Use of Technology (UTAUT) to develop a model to measure the usage and acceptance of storytelling in BI. The model was empirically evaluated through a survey.

Pfahlsberger, Mendling, and Eckhardt's research combines process mining and big data analytics capabilities. The authors use a design science research approach to develop a process mining alignment method for building big data analytics capabilities and evaluate it using expert interviews.

The final paper by Grønsund investigates algorithmic decision-making in an open data environment, seeking to understand the role of uncertainty. Based on the findings from a two-year long qualitative case study of an international maritime trade company, this research combines uncertainty and assemblage perspectives to gain a deeper understanding of the arrangements by which the case company configures relations of humans and machines.

We trust that you will find this year's selection of very diverse papers interesting, thought-provoking, and relevant for your research, education and/or professional practice. 